\title{
Le codage des mouvements volontaires par le cortex cérébral
}

L'exécution d'un mouvement, même très simple, implique de nombreuses opérations sensitivo-motrices, visuelles et cognitives, qui sont réalisées en moins d'une seconde. Ces opérations sont sous le contrôle de nombreuses régions corticales et sous-corticales, controlatérales au mouvement ou bilatérales. Il semble exister, de plus, indépendamment de la latéralité du mouvement, une spécialisation des hémisphères telle que l'hémisphère gauche (chez les droitiers) interviendrait particulièrement dans le contrôle des aspects cognitifs de l'action.

\section{Marc Jeannerod Jean-Paul Joseph}

\section{ADRESSE}

M. Jeannerod : Professeur des Universités. J.P. Joseph : Chercheur au Cnrs. Laboratoire de neuropsychologie expérimentale, Inserm U. 94, 16, avenue du Doyen-Lépine, 69500 a question posée dans cet article est celle du rôle du cortex dans la réalisation d'un mouvement volontaire. Pour y répondre, les aspects anatomiques, neurophysiologiques, comportementaux, cognitifs du contrôle moteur seront abordés. L'exécution d'un mouvement, même très simple en apparence (la prise manuelle d'un objet par exemple), implique en effet de nombreuses opérations: définition du but à atteindre, codage de la localisation de l'objet dans l'espace extra-corporel, sélection et coordination des membres et des segments concernés par l'action, adaptation de ces segments aux caractéristiques de l'objet, préparation posturale, codage de la vitesse et de la force des contractions musculaires, lecture des boucles de contrôle visuelles ou proprioceptives, etc. La plupart de ces opérations se déroulent pendant le temps relativement court (de l'ordre de quelques centaines de millisecondes) qui s'écoule entre le facteur déclenchant le mouvement et le début de son exécution. Il est donc plus que probable qu'elles ne s'enchaînent pas de manière séquentielle, mais qu'elles sont au contraire, pour une bonne part, réalisées simultanément grâce au fonctionnement parallèle de nombreuses structures nerveuses, en particulier au niveau cortical.

\section{Le réseau cortical en relation avec la production des mouvements volontaires}

Les techniques modernes d'imagerie cérébrale in vivo chez l'homme (comme la tomographie à émission de positons) permettent de se faire une idée du partage du travail entre les différentes régions du cortex lors de la production d'un mouvement [1]. Prenons comme exemple l'exécution par un sujet normal d'une séquence complexe de mouvements des doigts de la main droite. La tomographie à émission de positons (voir glossaire) réalisée sous inhalation d'oxygène 15 révèle chez ce sujet non seulement la présence d'une activation des aires motrice et somato-sensorielle primaires ( $\mathrm{Ml}$ et $\mathrm{Sl}$ ) de son hémisphère gauche, mais aussi une activation bilatérale de l'aire motrice supplémentaire (M2) et de l'aire prémotrice (PM), de l'aire somato-sensorielle secondaire (S2) et même de régions encore plus postérieures, comme le lobule pariétal supérieur. Au niveau sous-cortical, le noyau caudé, le putamen et le pallidum 
sont également activés des deux côtés. Si, dans la même expérience, la séquence motrice n'est qu’imaginée par le sujet au lieu d'être réellement exécutée, l'activation de $\mathrm{Ml}$ et Sl disparaît, tandis que l'activation bilatérale de M2 et de PM ainsi que celle des noỵaux gris reste clairement visible. Ces résultats permettent donc de distinguer la part d'activation du cortex qui revient à l'exécution proprement dite ( $\mathrm{Ml}$ et $\mathrm{Sl}$ ), de celle qui revient à sa préparation $[2,3]$.

La fonction de chacune des aires qui part icipent à ce réseau cortical est en partie révélée par l'étude de leurs connexions anatomiques (figure 1). Le cortex moteur primaire reçoit ses principales afférences corticales de $\mathrm{Sl}$ et de M2, et ses afférences sous-corticales des régions thalamiques connectées au cervelet. Les cellules d'origine du faisceau pyramidal, dont $60 \%$ sont situées dans Ml. se trouvent donc au point de rencontre d'informations concernant le programme à exécuter, venues de M2, et d'informations concernant son exécution ellemême, qui lui sont transmises par l'intermédiaire de $\mathrm{Sl}$ et des afférences cérebelleuses. Cette fonction d'exécution du cortex moteur primaire s'exerce, chez les primates, en priorité sur les motoneurones des groupes musculaires distaux (en particulier ceux de la main et des doigts). La section du faisceau pyramidal entraîne en effet une atteinte durable des mouvements fins et indépendants des doigts, comme ceux qui anticipent et accompagnent la manipulation d'objets $[4,5]$. Cette constatation corrobore le fait que les fibres du faisceau pyramidal se distribuent préférentiellement sur les motoneurones du groupe latéral, c'est-à-dire sur ceux qui innervent les muscles distaux.

M2 et PM, qui constituent (avec Ml) l'essentiel du cortex frontal agranulaire (voir glossaire), ont des connexions bien différentes de celles du cortex moteur primaire [6]. M2, qui contient une représentation somatotopique (voir glossaire) de l'ensemble du corps (moins précise toutefois que celle que contient $\mathrm{Ml}$ ), reçoit ses afférences du cortex somesthésique ( $\mathrm{Sl}$ et lobule pariétal supérieur), de $\mathrm{PM}$ et de la région dorsale du cortex pré-frontal, ainsi que de plusieurs noyaux thalamiques, dont ceux qui reçoivent les fibres en provenance des noyaux gris. La plupart de ces connexions sont réciproques. C'est donc par l'intermédiaire de Ml que M2 se trouve connecté avec l'appareil moteur périphérique. Sa contribution à la voie pyramidale est très discutée et ne concerne sans doute qu'une très faible proportion de cellules.

PM comprend en fait deux parties qui semblent fonctionnellement dis-

\section{${ }^{*}$ GLOSSAIRE*}

Archéocortex, paléocortex, néocortex: étapes de l'évolution phylogénétique du télencéphale chez les mammifères.

Cingulaire (cortex): zone du cortex qui appartient au système limbique. Outre le cortex cingulaire, le système limbique comprend les aires olfactives, l'hippocampe, le complexe amygdalien et l'aire septale. Cet ensemble, qui constitue le paléocortex des mammifères, intervient dans la régulation des émotions.

Granulaire (cortex) : la couche IV du cortex est constituée de petites cellules qui lui donnent un aspect plus ou moins granulaire. Cette couche IV est particulièrement développée dans les aires sensorielles du cortex (par exemple les aires visuelles) qui constituent, par extension, le cortex granulaire. Le cortex moteur, en revanche, est agranulaire, tandis que les régions préfrontales ont un aspect intermédiaire.

Somatotopie: représentation topographique des projections somesthésiques et proprioceptives sur le thalamus et le cortex cérébral. Par extension, on parle aussi de somatotopie pour désigner la projection topographique des régions motrices du cortex sur la musculature striée.

Tomographie à émission de positons: technique permettant de visualiser l'incorporation par les différentes régions du cerveau de molécules marquées par des isotopes radio-actifs émetteurs de positons. Ces isotopes, du fait de leur demi-vie très courte, doivent être préparés par un cyclotron situé à proximité immédiate du système de tomograhie. tinctes. La partie dorsale, qui jouxte M2, contient une représentation somatotopique limitée au tronc, tandis que la partie ventrale contient une représentation de la face, de la tête et du cou. Dans l'ensemble, PM reçoit ses afférences d'aires corticales associatives (cortex préfrontal, pariétal postérieur, préstrié) et entretient d'étroites connexions réciproques avec Ml et M2. PM ne contribue pas à la voie pyramidale, mais sa partie dorsale se projette massivement sur des régions du tronc cérébral d'où sont issues des voies qui contrôlent la musculature axiale et proximale. Cette organisation des connexions de M2 et de PM s'intègre dans un modèle de développement phylogénétique du néocortex qui considère l'existence de deux systèmes parallèles. Le premier système (dit système "dorsal » tirerait son origine de l'archéocortex (voir glossaire) (hippocampe) et engloberait le cortex cingulaire, les aires somatosensorielles, la partie médiane des lobes frontaux, incluant par conséquent les régions précentrales et prémotrices dorsales. Les corps striés feraient partie de cet ensemble. Le second système (dit système «ventral » tirerait son origine du paléocortex (voir glossaire) et engloberait l'insula, les autres aires sensorielles primaires et associatives, le cortex fronto-orbitaire et les aires précentrales et prémotrices ventrales [7, 8] (figure 1).

On peut tenter, à partir de ce modèle et sur la base d'expériences récentes chez le singe [9, 10] d'individualiser les fonctions respectives de $\mathrm{M} 2$ et de PM. M2 serait avant tout responsable du déclenchement de l'acte moteur, de sa dimension motivationnelle et de la préparation anticipée de l'organisme à ses conséquences, grâce à l'utilisation de programmes moteurs tenant compte des contraintes de l'appareil musculaire. Les lésions de l'aire motrice supplémentaire chez l'homme se traduisent essentiellement par une akinésie, caractérisée par une suspension transitoire de l'expression spontanée: rareté des mouvements des extrémités controlatérales à la lésion, immobilité du visage, absence d'expression orale [11].

A la différence de celles de M2, les fonctions de PM seraient plutôt liées 


\section{RÉFÉRENCES}

1. Roland PE, Skinhoj E, Lassen NA, Larsen B. Different cortical areas in man in organization of voluntary movements in extrapersonal space. J Neurophysiol 1980 ; 43 : 137-50.

2. Orgogozzo JM, Larsen B, Roland PE, Lassen NA. Activation de l'aire motrice supplémentaire au cours des mouvements volontaires chez l'homme. Rev Neurol (Paris) 1979 ; 135 : 705-17.

3. Roland PE, Friberg L. Localization of cortical areas activated by thinking. J Neurophysiol $1985 ; 53$ : 1219-43.

4. Chapman E, Wiesendanger M. Recovery of function following unilateral lesions of the bulbar pyramid in the monkey. Electroencephalogr Clin Neurophysiol 1982 ; 53 : 374-87.

5. Jeannerod $M$. The formation of finger grip during prehension. A cortically mediated visuomotor pattern. Behav Brain Res $1986 ; 19: 99-116$

6. Wiesendanger M. Organization of secondary motor areas of cerebral cortex. In : Brooks VB, ed. Motor control (part 2). The Neruous System. Handbook of Physiology. Bethesda: American Physiological Society, $1988,1121-47$

7. Sanides F. Functional architecture of motor and sensory cortices in primates in the light of a new concept of neocortex development. In : Noback CR, Montana W, eds. Advances in Primalology. Vol. I. New York: Appleton-Century Croft, 1971 : 137-208.

8. Pandya DN, Barnes CL. Architecture and connections of the frontal lobe. In: Perecman E, ed. The Frontal Lobes Revisited. New York: IRBN Press, 1987 : 41-72.

9. Passingham RE. Two cortical systems for directing movement. In : Motor Areas of the Cerebral Cortex. Ciba foundation Symposium $n^{\circ} 132$. Chichester: J. Wiley and Sons, $1987:$ 15l-61.

10. Rizzolatti G, Gentilucci M, Fogassi L, Luppino G, Matelli M, Ponzoni Maggi S. Neurons related to goal-directed motor acts in inferior area 6 of the macaque monkey. Exp. Brain Res 1987; 67 : 220-4.

11. Laplane D, Talairach J, Meininger V, Bancaud J, Bouchareine A. Motor consequences of motor area ablations in man. $J$ à l'utilisation d'informations extérieures au corps (en particulier visuelles) en vue de l'exécution des mouvements. C'est à ce niveau que serait codé le but de l'action en direction de l'environnement, qu'il s'agisse de ses dimensions temporelles (celles qui déterminent la séquence des mouvements) ou de caractéristiques appartenant en propre aux objets du monde extérieur. Les lésions de cette région sont le plus souvent, en clinique, associées à des lésions de Ml qui entraînent une hémiplégie masquant un éventuel déficit spécifique. Sa mise « hors circuit » par des lésions à distance affectant ses connexions afférentes, produisent des effets complexes qui seront discutés dans la partie intitulée : «La spécialisation hémisphérique pour la préparation et le contrôle des mouvements volontaire (page 490).

\section{L'intégration de l'action au comportement dirigé vers un but}

Ce schéma, nécessairement simplifié, du réseau cortical qui prend en charge la préparation et l'exécution des mouvements, doit être complété par des éléments qui assurent leur intégration à la finalité du comportement.

Le premier de ces éléments est le cortex pariétal postérieur, dont nous avons mentionné l'activation métabolique en relation avec la production de mouvements. Cette large zone du cortex comprend chez l'homme le lobule pariétal supérieur (aires 5 et 7 de Brodmann) et le lobule pariétal inférieur (aires 39 et 40). L'étude chez le singe des connexions cortico-corticales de régions qui peuvent être considérées comme homologues au cortex pariétal postérieur humain, révèle à ce niveau une large convergence d'informations en provenance des aires sensorielles somesthésiques et visuelles, des aires cingulaires et inféro-temporales, mais aussi de $\mathrm{Ml}$ et M2 et des régions préfrontales. En retour, le cortex pariétal postérieur se projette massivement sur le cortex préfrontal. L'activité des neurones de l'aire 7 chez le singe semble être liée aux aspects sensorimoteurs (et singulièrement visuomoteurs) du comportement. La décharge de ces neurones précède et/ou accompagne préféren- tiellement les mouvements de l'animal dirigés par des cibles visuelles «intéressantes» (des morceaux de nourriture, par exemple) situées dans l'espace extrapersonnel proche. D'autres neurones sont activés par les mouvements des yeux ou la présentation de stimuli visuels complexes [12].

L'implication du cortex pariétal postérieur dans le traitement des aspects spatiaux du mouvement est confirmée par les effets des lésions. Chez le singe, la lésion de l'aire 7 provoque une perte de la capacité de localiser et d'atteindre les objets avec la main controlatérale [13]. Chez l'homme, les effets des lésions, bien qu'ils diffèrent selon leur topographie au sein de la région pariétale postérieure et selon le côté atteint, vont dans le même sens. C'est ainsi qu'une lésion englobant le lobule pariétal supérieur provoque, quelque soit l'hémisphère atteint, un syndrome très voisin de celui de la lésion de l'aire 7 chez le singe (syndrome d'ataxie optique[14]). En revanche, une lésion du lobule pariétal inférieur entraîne, seulement lorsqu'elle est située à droite, un syndrome complexe traduisant l'incapacité du sujet à manipuler mentalement les données spatiales. De fait, les malades qui en sont atteints sont incapables de dessiner, surtout s'il s'agit d'un dessin comportant une perspective ; ils sont incapables de décrire verbalement un itinéraire familier, de dresser le plan de la pièce où ils se trouvent, de situer une ville sur une carte de géographie. Dans certains cas, la désorientation peut porter sur tout l'hémi-espace gauche (controlatéral à la lésion), que le sujet ignore dans son comportement et qu'il néglige dans la représentation qu'il se fait de son environnement (syndrome d'hémi-négligence spatiale) [15]. Cette spécialisation de l'hémisphère droit pour la représentation et l'utilisation des données spatiales repose sur une différenciation anatomique: la région pariétale postérieure de l'hémisphère droit est plus étendue que son homologue de l'hémisphère gauche, et possède une circonvolution supplémentaire [16]. L'intégration des mouvements dans le comportement est également assurée par l'activité du cortex préfrontal. On entend sous ce terme la partie la 


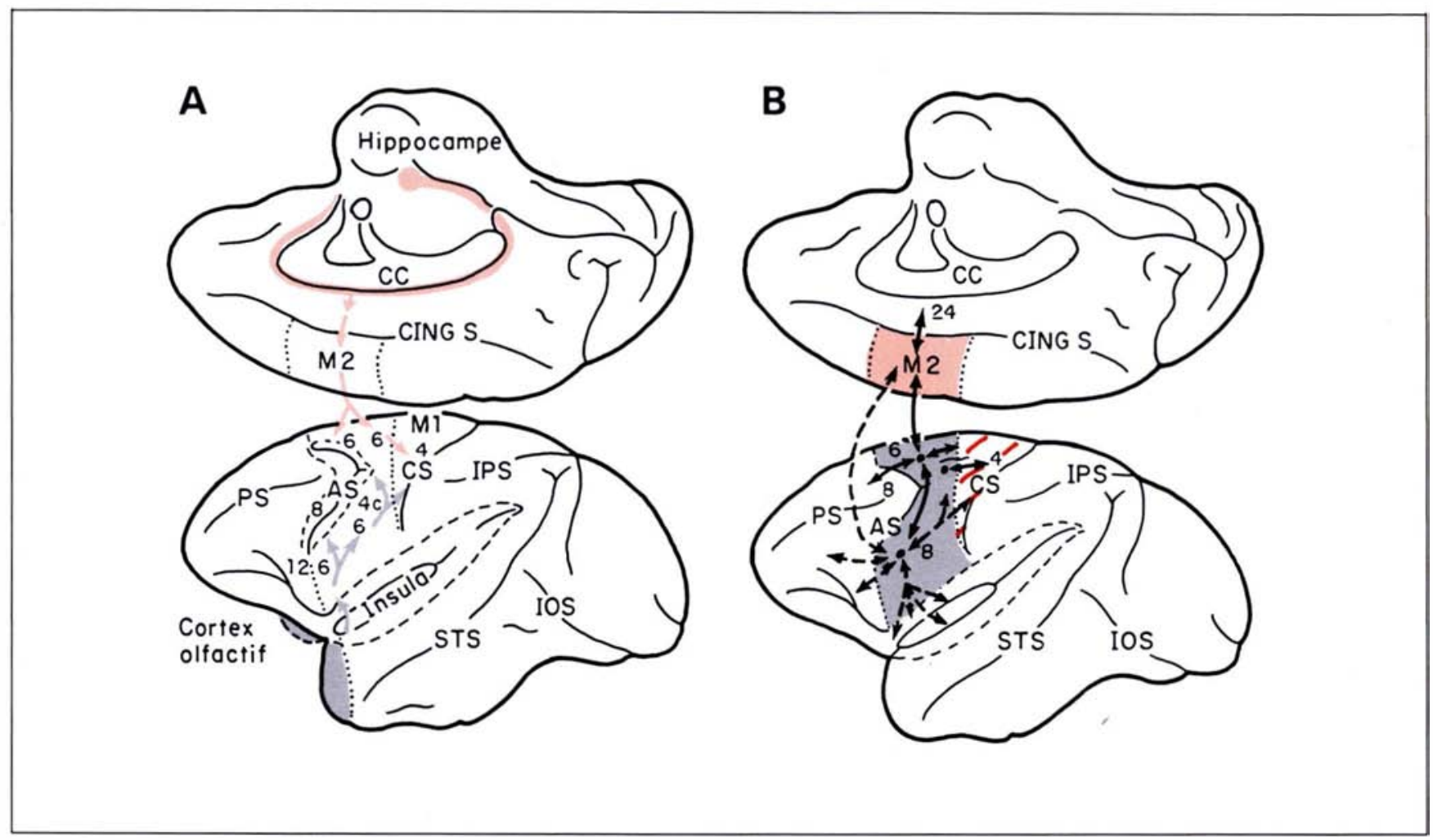

Figure 1. Topographie et connexions du cortex frontal chez le singe. La partie A représente la localisation des systèmes dorsal et ventral dont le développement phylogénétique a donné naissance respectivement aux parties dorsale et ventrale de l'aire 6 (cortex PM) et de l'aire 4 (cortex M1). L'aire motrice M2 occupe la partie médiane du système dorsal et est considérée comme une des étapes de la différenciation fonctionnelle de ce système. La partie $B$ représente les interconnexions de ces deux systèmes et leurs relations avec le cortex préfrontal (aires 8, 10 et 46 situées en avant du sillon arqué). Abréviations: $A S=$ sillon arqué ; $C C=$ corps calleux; $C F=$ scissure calcarine ; $C I N G S=$ sillon cingulaire ; $C S=$ sillon central ; IOS = sillon occipital inférieur ; IPS = sillon intrapariétal; PS = sillon principal; STS = sillon temporal supérieur. L'aire 24 correspond à la partie antérieure du cortex cingulaire (d'après [8]).

plus antérieure du cerveau, qui se définit anatomiquement chez tous les mammifères comme l'aire de projection corticale d'un noyau thalamique, le noyau médio-dorsal. Cette région subit chez les primates une expansion remarquable et constitue chez l'homme près de $30 \%$ de la surface du manteau cortical. Ses principales afférences corticales proviennent des aires associatives pariétales postérieures et inféro-temporales. La partie qui appartient au système «dorsal » reçoit en outre d'importantes afférences du cortex cingulaire, tandis que la partie qui appartient au système « ventral » est connectée à la région para-hippocampique ; ses efférences gagnent les aires M2, PM et les noyaux gris. Les connexions du cortex préfrontal avec le cortex pariétal et le cortex inférotemporal sont réciproques [8].

Les effets des lésions de cette région $\mathrm{m} / \mathrm{s} n^{\circ} 8 \mathrm{vol}$. t, octobre 88 se caractérisent, pour s'en tenir aux déficits «moteurs», par une incapacité du patient à se concentrer sur une tâche, du fait de la difficulté qu'il éprouve à inhiber des réponses motrices aux sollicitations de l'environnement. Ce déficit se traduit par une distractibilité anormale, des comportements de persévération motrice et une difficulté à planifier l'action en fonction d'un but un tant soit peu complexe ou lointain. Le malade devient en quelque sorte captif de son environnement.

Une série d'expériences portant sur les neurones de la région du sillon arqué chez le singe ont permis de comprendre certains des mécanismes préfrontaux assurant l'indépendance du comportement moteur vis-à-vis du monde extérieur. Joseph et Barone [17] ont montré que, lors de l'exécution d'une tâche motrice comportant des déplacements du regard, l'activation de certains de ces neurones dépendait à la fois de la présence d'un stimulus visuel dans une région particulière de l'espace et du fait que ce stimulus avait été désigné comme le but de l'action. De fait, ces neurones continuaient de décharger jusqu'au moment où le but de l'action pouvait être atteint, même si d'autres mouvement devaient être exécutés entre-temps. Dans une expérience similaire, la tâche de l'animal consistait à mémoriser l'ordre d'allumage de trois cibles localisées à des endroits différents, puis à les éteindre avec la main, dans le même ordre. L'animal devait donc planifier une séquence de mouvements des yeux et l'exécuter de mémoire. L'enregistrement de l'activité électrique de neurones de la région du sillon arqué au cours de cette tâche motrice a permis d'identifier deux populations neuronales, une qui code, avant exécution, 


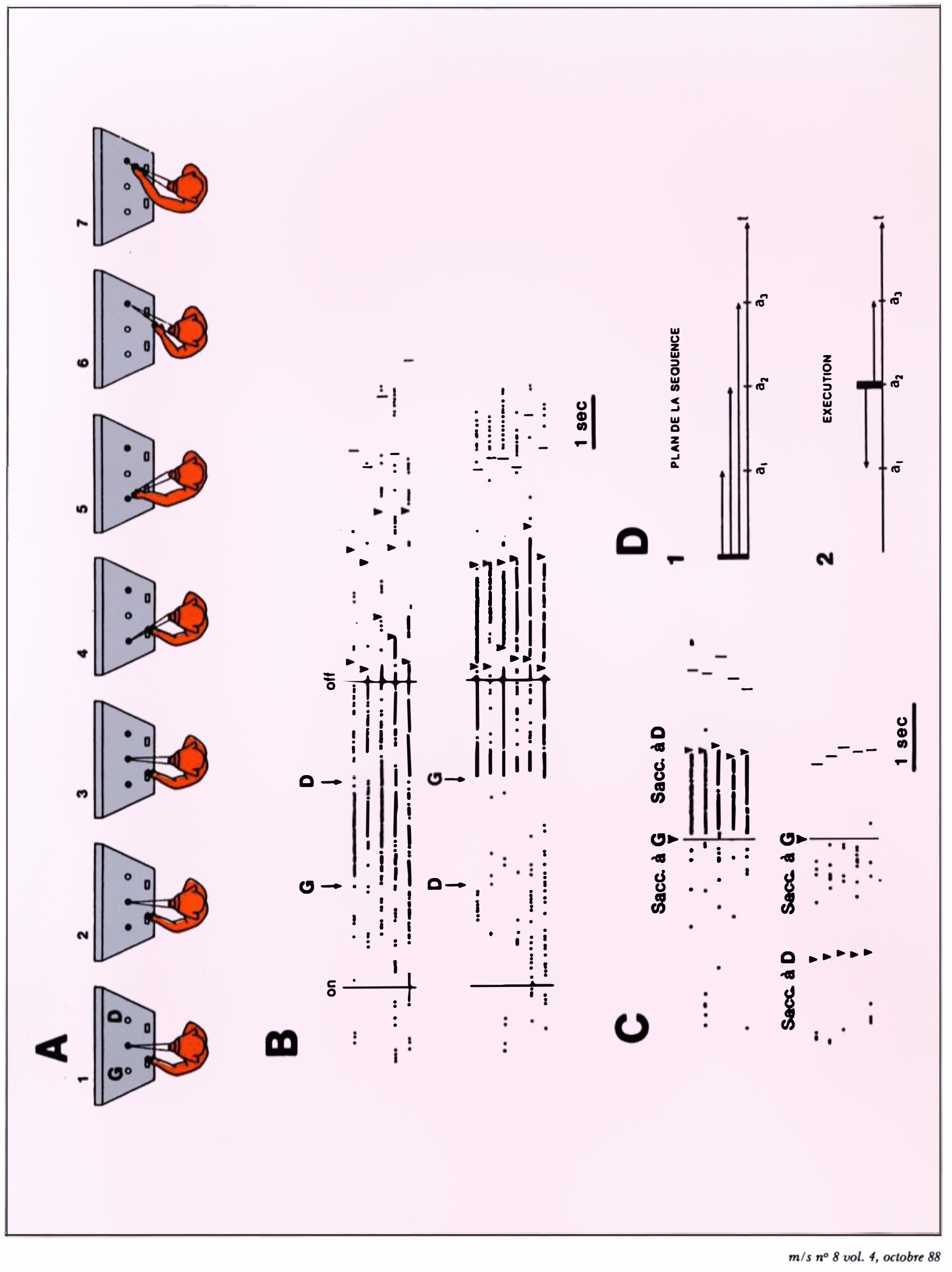


4 Figure 2. Caractéristiques de deux populations de neurones de la région du sillon arqué (aire 8) qui ont, chez le singe, un rôle important dans l'organisation de séquences oculo-motrices. A. La tâche consistait pour l'animal à stocker l'ordre d'apparition, variable d'un essai à l'autre, de deux cibles lumineuses latérales (D et G), tandis qu'il fixait du regard une cible lumineuse centrale (1). Sur ce diagramme la cible G apparait d'abord(2) suivie de la cible D(3). Quand le point de fixation central s'éteignait(4), l'animal devait porter son regard vers les cibles latérales et les éteindre successivement, par un mouvement du bras, dans l'ordre où elles avaient été allumées (5 à 7). B. Exemple d'un neurone qui stocke la position d'une des 2 cibles. Chaque ligne de points représente la décharge des potentiels d'action pendant un essai. Chaque essai débute par l'allumage du point central (on) et comporte successivement l'allumage des cibles $G$ et $D$, l'extinction du point central (off), le début de la saccade oculaire d'orientation vers la première, puis vers la deuxième cible (triangles inversés) et enfin le moment où la récompense est donnée à l'animal (barres verticales à la fin de chaque ligne). En général, l'animal atteint la première cible juste avant de s'orienter vers la seconde; l'apparition de la récompense coïncide avec l'extinction de la deuxième cible. Sur les 5 premiers essais (en haut), on constate que le taux de décharge de cette cellule est augmenté à l'apparition de la cible $G$ et que l'orientation du regard vers cette cible rétablit le taux de décharge à sa valeur de repos. Dans les 6 autres essais (au-dessous), la cible droite est allumée la première, puis la cible gauche. Le neurone est à nouveau activé par l'apparition de la cible gauche. Ni la fixation de l'autre cible (D), ni la préparation d'un mouvement du bras vers cette cible ne modifient le taux de décharge de la cellule. Ce résultat démontre que ce neurone joue un rôle dans le stockage de la position spatiale d'un objet que l'animal utilisera ultérieurement dans la séquence comportementale. Certains neurones codent également pour les relations temporelles entre les mouvements dirigés vers différents objets: la position d'un objet n'est alors mémorisée que si elle est dans une certaine relation temporelle avec celle des autres objets. C. Exemple d'un neurone qui code pour le contexte spatial et temporel d'une tâche en cours. Dans les deux diagrammes, les essais sont alignés sur le début des saccades oculaires vers la cible G. Lors des 5 premiers essais (en haut), l'orientation vers la cible $G$ et la fixation de cette cible précèdent l'orientation vers la cible $D$. Dans les 5 essais suivants (en bas), elle la suit. On constate que le neurone n'est activé lors de la fixation de la cible $G$ que si la cible $D$ est encore allumée. Ce neurone n'est donc pas un neurone de fixation. On peut montrer sur d'autres exemples qu'il ne code pas non plus la préparation de la saccade vers la cible $D$. II code pour le contexte spatial (* cible $D$ encore allumée $/$ de la fixation en cours. L'activité tonique de ce type de neurones pourrait avoir pour rôle de créer une représentation de la prochaine cible vers laquelle l'animal va s'orienter. Certains neurones codent également dans les mêmes conditions l'information «cible $D$ déjà éteinte *. D. Représentation schématique des deux étapes principales, individualisées en terme de populations neuronales, de l'exécution d'une série d'actes moteurs dirigés vers différents points mémorisés de l'espace. On considère une séquence de trois tâches successives, a1, a2, a3. 1. Avant l'exécution de la tâche, une première population de neurones stocke, sous la forme d'une activité tonique, la position des différentes régions, ainsi que leurs relations temporelles dans la séquence. Cette population code par conséquent le plan général de la séquence. 2. Pendant l'exécution de la tâche, la deuxième population de neurones génère à chaque étape, une représentation de la région de l'espace vers laquelle devra être exécuté le prochain mouvement. La formation de cette représentation résulte d'une intégration de données relatives aux positions des tâches déjà exécutées, de celles qui restent à exécuter et de la tâche en cours. Ces neurones prennent en charge l'exécution pas-à-pas de la séquence. (Barone et Joseph, en cours de publication.)

pour le plan de la séquence et une autre qui code, pendant l'exécution, pour l'état de la séquence, c'est-à-dire la position des cibles qui sont éteintes et de celles qui restent allumées. Les propriétés de la seconde population suggérent qu'à chaque étape du trajet oculomoteur, l'animal bâtit une représentation de la région de l'espace où se trouve la prochaine cible. Ces représentations, concernant les objets et leur situation par rapport au corps, pourraient être élaborées dans les régions pariétales postérieures et inféro-temporales et substituées dans les boucles sensorimotrices aux informations directement fournies par les organes des sens. Ces deux propriétés - possibilité de construire des représentations mémorisées du monde extérieur et de les utiliser comme but d'une action - semblent caractériser la fonction préfrontale et rendre compte, pour l'essentiel, des mécanismes qui assurent l'autonomie relative du sujet par rapport à l'environnement (figure 2).

\section{Contrôles intra- et inter-hémisphériques des mécanismes visuo-moteurs}

Il existe des situations où le mouvement dirigé vers une cible localisée dans l'espace extérieur requiert en principe la collaboration des deux hémisphères : c'est le cas lorsqu'une main est utilisée pour atteindre un objet présenté dans le champ visuel du côté opposé, et où l'information visuelle traitée par l'hémisphère qui « voit » l'objet doit pouvoir être accessible à celui qui commande la réponse motrice. Cette affirmation toutefois s'inscrit dans la logique d'un contrôle exclusif des mouvements par des voies croisées, maintenant largement remise en cause. Des observations expérimentales et cliniques réalisées après blocage du transfert inter-hémisphérique (par section du corps calleux, par exemple) ont en effet démontré l'existence d'une contribution de voies motrices ipsilatérales (non croisées) au contrôle visuo-moteur.

On peut rappeler à ce sujet les classiques expériences chez le singe à cerveau dédoublé, par section du corps calleux et du chiasma optique. De tels animaux, lorsqu'ils utilisent la main ipsilatérale à l'œil stimulé, sont bien capables d'atteindre la position du morceau de nourriture qui leur est présenté, mais sont par contre incapables de le saisir et de le manipuler [18].

Chez l'homme, la section chirurgicale du corps calleux ou les lésions interrompant le transfert inter-hémisphérique entraînent des déficits caractéristiques des mouvements des doigts. Pour tester les effets de ces lésions, on demande aux malades d'imiter avec leur propre main des postures digitales qui leur sont présentées sous la forme de dessins. La présentation est faite de manière très 


\section{RÉFÉRENCES}

12. Hyvärinen J. The parietal cortex of monkey and man. Berlin: Springer-Verlag, 1982 : 202.

13. Faugier-Grimaud S, Frenois C, Stein DG. Effects of posterior parietal lesions on visually guided behavior in monkeys. Neuropsychologia 1978; 16 : 151-68.

14. Perenin MT, Vighetto A. Optic ataxia : a specific disorder in visuomotor coordination. In : Hein A, Jeannerod M, eds. Spatially Oriented Behavior. New York: Springer, 1983 : 305-26.

15. Jeannerod M. Neurophysiological and neuropsychological aspects of spatial neglect. Amsterdam : North-Holland, 1987.

16. Eidelberg D, Galaburda AM. Inferior parietal lobule. Divergent architectonic asymmetries in the humain brain. Arch Neurol $1984 ; 41: 843-52$.

17. Joseph JP, Barone P. Prefrontal unit activity during a delayed oculomotor task in the monkey. Exp Brain Res 1987 ; 67 : 460-8.

18. Brinkman J, Kuypers HG JM. Cerebral control of contralateral and ipsilateral arm, hand and finger movements in the split-brain rhesus monkey. Brain 1973 ; 96 : 653-74.

19. Gazzaniga MS, Bogen JE, Sperry RW. Dyspraxia following division of the cerebral commissures. Arch. Neurol 1967 ; 16:606-12.

20. Jeannerod $\mathbf{M}$. The neural and behavioural organization of goal-directed movements. Oxford : Oxford University Press, 1988.

21. Signoret JL, North P. Les apraxies gestuelles (apraxie idéatoire, apraxie idéomotrice, apraxie motrice). Rapport de neurologie, Congrès de psychiatrie et de neurologie de langue française, $77^{e}$ session, Angers. Paris : Masson, 1979.

22. Geschwind N. Disconnection syndromes in animals and man. Brain $1965 ; 88$ : 237-94, 585-644.

23. Jeannerod M. Geste et langage. La contribution de Henry Hécaen au problème des fonctions de l'hémisphère gauche. Evol Psy- brève dans une moitié du champ visuel, de façon à ne stimuler qu'un seul hémisphère. L'imitation est correcte lorsqu'elle est faite avec la main controlatérale à l'hémisphère stimulé, mais échoue si elle doit être faite avec la main ipsilatérale [19]. Ces résultats, tant des expériences chez le singe que des observations cliniques chez l'homme, confirment le rôle prédominant de la voie corticospinale croisée dans le contrôle des mouvements fins des a'oigts (que démontraient déjà les effets des lésions du faisceau pyramidal). Le contrôle des mouvements proximaux, par contre, est plus complexe : il s'exerce non seulement par l'intermédiaire de la voie croisée comme pour les mouvements distaux, mais aussi par l'intermédiaire de voies non croisées. Ces voies sont en grande partie issues du tronc cérébral, et sont elles-mêmes sous le contrôle des aires motrices non primaires, en particulier PM. Ce schéma anatomique permet d'expliquer l'aspect, déjà évoqué dans le premier sous-chapitre (page 484), de l'activation métabolique du cortex lors de l'exécution d'un mouvement complexe de la main. Alors que seule l'aire $\mathrm{Ml}$ controlatérale au mouvement est activée (ce qui traduit la mise en jeu des muscles distaux par la voie pyramidale croisée), les aires M2 et PM des deux côtés sont activées (ce qui traduit la mise en jeu relativement diffuse et bilatérale des muscles posturaux par les voies ipsilatérales).

Cette dualité du système moteur peut être mise en rapport avec la dualité des fonctions motrices inhérente à tout mouvement dirigé vers un but à l'extérieur du corps. La fonction de localisation du but par rapport au corps, qui consiste à atteindre un point de l'espace, fait intervenir les muscles proximaux, tandis que la fonction d'identification, qui consiste à saisir ou à manipuler, fait intervenir les muscles distaux [20].

\section{La spécialisation hémisphérique pour la préparation et le contrôle des mouvements volontaires}

Certains aspects du contrôle des mouvements semblent relever d'une spécialisation hémisphérique. Ce pro- cessus de latéralisation fonctionnelle, dont les exemples type sont la spécialisation de l'hémisphère gauche pour le langage et celle de l'hémisphère droit pour la représentation de l'espace, concerne donc avant tout les fonctions dites « cognitives». Dans le domaine du mouvement volontaire, ces fonctions recouvrent des opérations telles que l'intention et la représentation du but à atteindre, la décision d'agir, l'analyse du contexte dans lequel se déroule l'action, la détermination du contenu émotionnel et communicationnel des mouvements. Les premières données suggérant une participation préférentielle de l'hémisphère gauche à l'organisation du comportement gestuel datent du début du siècle. Il avait alors été constaté que certains sujets porteurs d'une lésion de l'hémisphère gauche (et souvent d'une hémiplégie droite), présentaient un trouble curieux des gestes de la main gauche, qualifié par Liepmann d'«apraxie»[21]. Des lésions de zones homologues de l'hémisphère droit n'entraînaient pas de semblables effets. Les malades apraxiques, tels qu'ils peuvent être couramment observés, sont incapables d'exécuter des mouvements expressifs ou symboliques (le salut militaire), ni de mouvements consistant à mimer l'utilisation d'un objet (se brosser les dents) ; ils ne peuvent reproduire par imitation des mouvements simples exécutés par l'examinateur; ils ne peuvent réaliser à l'aide d'objets réels, d'actions comportant une séquence un tant soit peu complexe (plier une lettre et la mettre sous enveloppe). L'interprétation que l'on peut donner de ce classique syndrome (l'apraxie idéomotrice) est qu'il existerait à gauche (chez le sujet droitier) une structure d'ordre hiérarchique plus élevé que les aires motrices d'exécution, qu'elle tiendrait sous sa dépendance. Une lésion de cette région entraînerait donc automatiquement une apraxie bilatérale ou, lorsque la voie pyramidale issue du cortex moteur gauche serait également lésée, une apraxie gauche associée à une hémiplégie droite. Une lésion épargnant cette région mais détruisant ses connexions avec l'hémisphère droit entraînerait, quant à elle, une apraxie unilatérale gauche isolée. De fait, ce dernier cas est 
observé chez des malades présentant une lésion limitée au corps calleux, sans atteinte corticale [22].

L'association fréquente de l'apraxie avec une hémiplégie droite laisse penser que la région critique pourrait être plus ou moins confondue avecles aires prémotrices (M2 et/ou PM) de l'hémisphère gauche. Toutefois, les troubles provoqués par des lésions localisées à ce niveau concernent l'exécution proprement dite des mouvements, plutôt que l'organisation de l'action. Les lésions corticales responsables de l'apraxie sont en fait pratiquement toujours situées dans la région pariétale postérieure gauche. C'est donc dans la rupture des connexions entre les aires associatives pariétales et le cortex frontal que doit être recherchée l'explication de l'apraxie.

Le rôle de l'hémisphère gauche dans le contrôle des aspects cognitifs de l'action soulève le problème de la parenté des mécanismes contrôlant respectivement le geste et le langage [123]. D'intéressantes observations ont été faites chez des sourdsmuets congénitaux atteints de lésions hémisphériques. Ces sujets utilisent normalement pour communiquer un langage gestuel possédant une structure formelle de même nature que les langues parlées. Lorsque la lésion est située du côté gauche, ils présentent une véritable «aphasie gestuelle», leurs erreurs portant à la fois sur la morphologie des gestes (qui sont mal «articulés») et sur le lexique. On peut bien penser, dans ce cas, que le trouble gestuel est directement lié à l'atteinte du système de signes utilisé pour la communication, au même titre que dans l'aphasie verbale et dans l'apraxie.

Le réseau cortical responsable du mouvement volontaire comporte donc plusieurs niveaux de traitement, en quelque sorte emboités les uns dans les autres, à chacun desquels correspond un mode particulier de représentation de l'action. Le niveau sensori-moteur réalise l'adéquation des mouvements à la forme et à la localisation spatiale des objets. Le niveau motivationnel code pour le contexte de l'action et la valeur affective des stimuli. Enfin, le niveau symbolique élabore la séquence des gestes et leur donne leur signification

\section{Summary}

The cortical mechanisms involved in voluntary movements are reviewed. Several different cortical areas are considered. The primary motor cortex $(\mathrm{Ml})$, which receives peripheral inputs from the primary somato-sensory area and from the cerebellum via thalamic nuclei, is primarily involved in the execution of proximal and distal contralateral limb movements via the pyramidal tract. The secondary motor area (SMA or M2) and the dorsal part of the premotor cortex (PM) receive inputs from associative cortex and exert their action via $\mathrm{Ml}$ and extra-pyramidal motor pathways. These areas are implicated in action preparation in developing preparatory states of the musculature that anticipate the effects of movement execution. In addition, the lateral part of PM is related to the organization of actions based on the value or affective significance of the triggering stimuli. The role of prefrontal cortex is to construct memory-based representations of the external world which will substitute in the sensory-motor loops to sensory information provided by the sense organs. Within the parietal cortex, an image of the body and its parts in relation to external space is constructed. This image is used to guide the body and its parts - the eyes and the limbs - to targets of interest in space.

\section{TIRÉS A PART}

M. Jeannerod. 